\title{
Adaptive Storage Battery Management based on the Energy on Demand Protocol
}

\author{
Takekazu Kato \\ Academic Center for Computing and \\ Media Studies, Kyoto University \\ Kyoto, Japan \\ t.kato@ieee.org
}

\author{
Kento Tamura \\ Graduate School of Informatics \\ Kyoto University \\ Kyoto, Japan \\ ktamura@vision.kuee.kyoto-u.ac.jp
}

\author{
Takashi Matsuyama \\ Graduate School of Informatics \\ Kyoto University \\ Kyoto, Japan \\ tm@i.kyoto-u.ac.jp
}

\begin{abstract}
We proposed the concept of i-Energy[1] as a novel smart demand-side energy management scheme to realize efficient and versatile control of e-power flows among decentralized energy generation/storage devices and appliances in homes, offices, and neighboring communities. To embody the concept, we developed a novel energy management method named Energy on Demand (EoD) for a single power source[3]. This paper proposes an adaptive storage battery management method based on EoD. The novelty of the method rests in the demand-based battery capacity design and dynamic charge/discharge control. The effectiveness of the proposed method has been proved with experiments using real life data in the smart apartment room [5].
\end{abstract}

Keywords-component; i-Energy; Energy on Demand; storage battery management; home energy management system (HEMS)

\section{INTRODUCTION}

The essential task of electrical power network management systems is to keep the balance between power supplies and demands. The difficulties of the task are best characterized by dynamically changing power supplies and demands; power outputs of some power supply devices, e.g. PVs, fluctuate dynamically and power consumption patterns change continuously depending on personal and social activities.

In Smart-Grid systems, demand-response and dynamic pricing mechanisms[2] are introduced to curb peak power demands down below the maximum possible power supply. While their effectiveness has been demonstrated, their power curbing capabilities are limited; (1) the power reduction rate is limited to about $10-15 \%$, (2) consumers should make efforts everyday to respond to supplier's requests, and (3) real-time demand curbing is not possible by human efforts.

To solve these problems, the development of smart demandside energy management systems is required, which automatically respond to supplier's requests. Moreover, with the introduction of distributed power devices such as PVs, wind power generators, and storage batteries into demand sides, autonomous self-contained energy management, i.e. power- balancing, capabilities should be implemented in demand-side energy management systems.

In [3], we proposed a novel demand-side energy management system, named Energy on Demand (EoD) system, where the power is not supplied immediately when an appliance is switched on, instead a power request message is issued to a home energy manager. Then, the manager mediates such requests taking into account available energy sources as well as appliance priorities. This mediation is conducted based on the "best effort" policy; some demands with low priorities may not be satisfied. Moreover, one can set a ceiling value for the power supply; the manager supplies power only when the ceiling is satisfied. This ceiling control guarantees the reduction rate of power consumption as well as realizes the real-time response to supplier's power curbing requests.

In the previous work[3], we developed the EoD system for a single commercial utility power source, and proposed a demand mediation algorithm. We augment the EoD system so that it can manage multiple power sources. This paper especially describes the case of two power sources, which a storage battery is added into the single power source system. The augmentation includes power-supplying planning and realtime demand mediation algorithms. The effectiveness of the augmented EoD system is demonstrated with experiments using real life data in the smart apartment room, where all appliances are equipped with smart taps [4] to monitor and control their power consumptions.

\section{OVERVIEW OF THE ENERGY ON DEMAND SYSTEM}

Fig. 1 illustrates a basic scheme of the EoD system, where the real-time power balancing is realized between smart appliances and smart power sources. Smart taps attached to the appliances enable the power manager to monitor and control power consumptions real time. Several types of smart taps have been developed depending on appliance power controllabilities: while power consumptions of light bulbs can be directly controlled, those of air conditioners can be controlled indirectly by changing operation modes and/or specifying temperatures. Power routers attached to distributed 


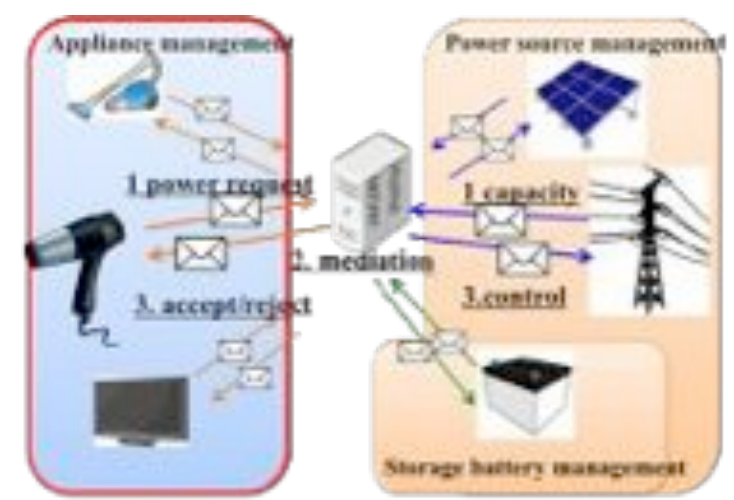

Fig. 1 Overview of the Energy on Demand System.

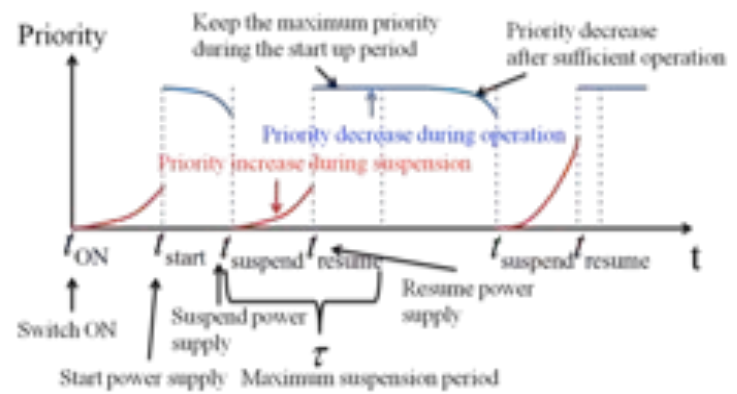

Fig. 2 Dynamic priority function for an air conditioner.

power devices enable the power manager to monitor and control power generations real time.

In principle, the EoD system operates as follows. 1) When one wants to use an appliance (i.e. switches on the appliance), the appliance, more specifically the smart tap attached to it, sends a "power request message" to the manager. 2) The manager decides the suppliable power taking into account the current total power consumption of running appliances, the capacity of the power source(s), and the priority of the appliance. 3) The manager returns a "power allocation or refusal message" to the requesting appliance. 4) Then, the allocated amount of electric power is supplied to the appliance.

For a commercial utility power source, we can specify its capacity by i) a "restriction" value to limit the instantaneously suppliable power to appliances to reduce the peak power and ii) a "ceiling" value to reduce the accumulated power consumption for a specified period of time, e.g., for a day or a month. The former enables the real time peak cut to cope with demand response requests from utility companies. The latter guarantees the reduction of the electricity cost and $\mathrm{CO}_{2}$ emission.

As for the system architecture, the EoD system consists of two major processes: the off-line planning and the real-time mediation processes. The former makes a power supply and consumption plan for a specified period based on pre-learned power consumption patterns, satisfying the user-specified restriction and ceiling values. The latter, which operates as described above, is implemented by three real-time processes: 1) the event-driven process mediates power requests from appliances to comply with the plan, 2) the watch-dog process monitors and curbs the total power consumption if it is expected to go beyond the planned restriction value due to power consumption fluctuations of appliances, and 3) the periodical re-planning process modifies the plan dynamically by feedbacking differences between the planned and actual power consumptions.

Another novel feature of the EoD system is the dynamic priority function defined for each appliance. That is, the appliance priority is dynamically changed according to appliance utilities and operation conditions. Fig. 2 illustrates an example of the priority change of an air conditioner. Even if switched ON, its operation can be delayed and the power supply can be suspended during operation. The priority increases during delay and suspension periods to start/resume operation, while the priority decreases after sufficient operation.

The demand mediation algorithm for the EoD system with a single commercial utility power source is formulated in [3]. In what follows, we augment the algorithm to cope with a storage battery and realize the demand-based battery capacity design and dynamic charge/discharge control.

\section{AUGMENTED EOD SYSTEM WITH A STORAGE BATTERY}

\section{A. Power Supply and Consumption Planning}

Suppose we want to make a power supply and consumption plan for a day. The planning process first estimates $\mathrm{PD}_{a}(t)$, the power consumption pattern of each appliance $a \in A$ (set of appliances) based on its pre-learned power consumption patterns.

Let $\operatorname{PS}_{S}(t)$ be a power supply pattern of power source $s \in\{c, b\}$, where $c$ and $b$ denote the commercial utility power source and storage battery respectively. Then, the power balance constraint between supplies and demands can be represented by the following equation.

$$
\sum_{a \in A} \mathrm{PD}_{\mathrm{a}}(t)=\sum_{s \in\{c, b\}} \mathrm{PS}_{s}(t) .
$$

Note here that $\mathrm{PS}_{b}(t)>0$ implies discharge, i.e. power supply to appliances while $\mathrm{PS}_{b}(t)<0$ charge from $\operatorname{PS}_{c}(t)$.

The accumulated power supply from the utility power source $\mathrm{ES}_{c}(t)$ and the accumulated power in the battery $\mathrm{ES}_{b}(t)$ can be defined as follows:

$$
\begin{gathered}
\mathrm{ES}_{c}(t)=\sum_{\delta=0}^{t} \operatorname{PS}_{c}(\delta) \\
\mathrm{ES}_{b}(t)=\sum_{\delta=0}^{t} \operatorname{PS}_{\mathrm{b}}^{\prime}(\delta) \\
\operatorname{PS}_{b}^{\prime}(t)=\left\{\begin{array}{l}
-\alpha_{b} \mathrm{PS}_{b}(t) \quad \text { if } P S_{b}(t)<0 \\
-\frac{1}{\alpha_{b}^{\prime}} \mathrm{PS}_{b}(t) \quad \text { otherwise }
\end{array}\right.
\end{gathered}
$$

where $0<\alpha_{b}<1$ and $0<\alpha_{b}^{\prime}<1$ denote charge and discharge efficiencies respectively. Note that all algorithms presented in this paper compute accumulated powers based on short-term discretized ones. Thus Eq. (2) and (3) above are defined with summations rather than integrals. 
As described before, a human manager can set the restriction $\overline{\mathrm{PS}_{c}(t)}$, the maximum instantaneous power supply, and the ceiling $\overline{\mathrm{ES}_{c}}$, the maximum accumulated power supply, for the utility power source. The storage battery, on the other hand, is characterized with the maximum discharge power $\overline{\mathrm{PS}_{b}}$, the maximum charge power $-\mathrm{PS}_{b}$, and the capacity of storable power $\overline{\mathrm{ES}_{b}}$. Then, each power source should comply with the following constraints:

$$
\begin{gathered}
\mathrm{PS}_{c}(t)<\overline{\mathrm{PS}_{c}(t)}, \mathrm{ES}_{c}(T)<\overline{\mathrm{ES}_{c} .} \\
\underline{\mathrm{PS}_{b}}<\mathrm{PS}_{b}(t)<\overline{\mathrm{PS}_{b}}, 0<\mathrm{ES}_{b}(t)<\overline{\mathrm{ES}_{b}},
\end{gathered}
$$

where $T$ denotes the length of the planning period, e.g. 24 hours for the daily planning. While not implemented yet, more sophisticated battery characterizations such as natural discharing and hysteresis effects can be represented by introducing additional constraints on $E S_{b}(t)$.

1) Initial plan to satisfy the restriction and the ceiling

The initial power usage plan $\operatorname{PD}^{\mathrm{PLAN}}(t)$ is generated to satisfy Eq.(5) as follows:

$$
\begin{gathered}
\operatorname{PD}^{\mathrm{PLAN}}(t)=\frac{\overline{\mathrm{ES}_{c}}}{\sum \mathcal{D}^{\prime}(t)} \mathcal{D}^{\prime}(t), \\
\mathcal{D}^{\prime}(t)=\left\{\begin{array}{cc}
\overline{\operatorname{PS}_{c}(t)} & \text { if } \mathcal{D}(t)>\overline{\operatorname{PS}_{c}(t)} \\
\mathcal{D}(t) & \text { otherwise }
\end{array}\right.
\end{gathered}
$$

where $\mathcal{D}(t)=\sum_{a \in A} \operatorname{PD}_{\mathrm{a}}(t)$.

2) Improve the initial plan to minimize user's demand dissatisfaction

Since $\operatorname{PD}^{\text {PLAN }}(t)$ restricts power demand peaks below $\overline{\mathrm{PS}_{c}(t)}$ always, a user may feel uncomfortable when he/she really needs big powers to do some everyday life activities like cooking. The introduction of a battery can solve this problem. That is, with a battery, $\mathrm{PD}^{\mathrm{PLAN}}(t)$ can be improved to reduce user's demand dissatisfaction while keeping the restriction and ceiling constraints.

First, we define the dissatisfaction function DS(PD $\left.{ }^{\text {PLAN }}\right)$ for the power usage plan $\operatorname{PD}^{\mathrm{PLAN}}(t)$ as follows:

$$
\operatorname{DS}\left(\operatorname{PD}^{\mathrm{PLAN}}\right)=\frac{1}{|T|} \sqrt{\sum_{t=0}^{T}\left(\mathcal{D}(\mathrm{t})-\operatorname{PD}^{\mathrm{PLAN}}(t)\right)^{2}} .
$$

Then, the problem of improving the initial plan to minimize user's demand dissatisfaction can be defined as follows:

minimize: DS(PD $\left.{ }^{\text {PLAN }}\right)$

subject to : $\mathrm{PS}_{c}^{\mathrm{PLAN}}(\mathrm{t})<\overline{\mathrm{PS}_{c}(t)}, \mathrm{ES}_{c}^{\mathrm{PLAN}}(T)<\overline{\mathrm{ES}_{c}}$

$$
\begin{gathered}
\underline{\mathrm{PS}_{b}<} \mathrm{PS}_{b}^{\mathrm{PLAN}}(t)<\overline{\mathrm{PS}_{b}}, 0<\mathrm{ES}_{b}^{\mathrm{PLAN}}(t)<\overline{\mathrm{ES}_{b}} \\
\operatorname{PD}^{\mathrm{PLAN}}(t)=\operatorname{PS}_{c}^{\mathrm{PLAN}}(t)+\operatorname{PS}_{b}^{\mathrm{PLAN}}(t),
\end{gathered}
$$

where the superfix "PLAN" implies the functions are defined in the power usage plan.

We developed an iterative algorithm for this minimization problem. The basic idea is to iteratively modify $\operatorname{PD}^{\mathrm{PLAN}}(t)$, $\operatorname{PS}_{\mathrm{c}}^{\mathrm{PLAN}}(t)$, and $\mathrm{PS}_{b}^{\mathrm{PLAN}}(\mathrm{t})$ to reduce user's demand

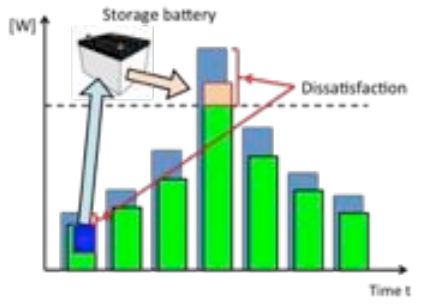

Fig. 3 Minimizing user's demand dissatisfaction by time-shifting power consumptions with a battery.

dissatisfaction by time-shifting power consumptions with a battery (Fig. 3). The computational algorithm is defined as follows.

\section{[Initialize]}

Compute PD ${ }^{\text {PLAN }}(t)$ by Eqs. (7) and (8) and set $\operatorname{PS}_{\mathrm{C}}^{\mathrm{PLAN}}(t)=\operatorname{PD}^{\mathrm{PLAN}}(t)$ and $\operatorname{PS}_{b}^{\mathrm{PLAN}}(t)=0$. Let $\mathcal{T}_{\mathrm{DST}}=\left\{t \mid 0 \leq t \leq T, \mathcal{D}(t)-\mathrm{PD}^{\mathrm{PLAN}}(t)>0\right\}$ denote the set of discretized short-term temporal units where user's demands are not fully satisfied.

[Step 1.]

Select the maximum dissatisfaction time $t_{\text {DST }}$ from $\mathcal{T}_{\text {DST }}$.

$$
t_{\mathrm{DST}}=\underset{t \in \mathcal{T}_{\mathrm{DST}}}{\arg \max }\left(\mathcal{D}(t)-\mathrm{PD}^{\mathrm{PLAN}}(t)\right)^{2}
$$

Then compute $\mathcal{T}_{\mathrm{SRC}}=\left\{t \mid 0 \leq t<t_{\mathrm{DST}}\right\}$.

[Step 2.]

Select the minimum dissatisfaction time $t_{\text {SRC }}$ from $\mathcal{T}_{\text {SRC }}$.

$$
\begin{gathered}
t_{\mathrm{SRC}}=\underset{t \in \mathcal{T}_{\mathrm{SRC}}}{\arg \min }\left(\mathcal{D}(t)-\mathrm{PD}^{\mathrm{PLAN}}(t)\right)^{2} \\
\text { If }\left(\mathcal{D}\left(t_{\mathrm{SRC}}\right)-\mathrm{PD}^{\mathrm{PLAN}}\left(t_{\mathrm{SRC}}\right)\right)^{2}<\left(\mathcal{D}\left(t_{\mathrm{DST}}\right)-\mathrm{PD}^{\mathrm{PLAN}}\left(t_{\mathrm{DST}}\right)\right)^{2},
\end{gathered}
$$

then go to Step 3. Otherwise, go to Step. 4.

\section{[Step 3.] Battery charge/discharge planning}

Charging: Decrease $\mathrm{PD}^{\mathrm{PLAN}}\left(t_{\mathrm{SRC}}\right)$ and decrease $\operatorname{PS}_{b}^{\mathrm{PLAN}}\left(t_{\mathrm{SRC}}\right)$, i.e. charge the battery, by $C$ at $t_{\mathrm{SRC}}$, while keeping $\operatorname{PS}_{c}^{\text {PLAN }}\left(t_{\text {SRC }}\right)$ (see Eq.(13)),

$$
\begin{aligned}
& \operatorname{PS}_{b}^{\text {PLAN }}\left(t_{\mathrm{SRC}}\right)=\operatorname{PS}_{b}^{\mathrm{PLAN}}\left(t_{\mathrm{SRC}}\right)-C, \\
& \operatorname{PD}^{\mathrm{PLAN}}\left(t_{\mathrm{SRC}}\right)=\operatorname{PD}^{\mathrm{PLAN}}\left(t_{\mathrm{SRC}}\right)-C .
\end{aligned}
$$

Discharging: Increase $\operatorname{PD}^{\mathrm{PLAN}}\left(t_{\mathrm{DST}}\right)$ and increase $\operatorname{PS}_{b}^{\text {PLAN }}\left(t_{\mathrm{DST}}\right)$, i.e. discharge the battery, at $t_{\mathrm{DST}}$,

$$
\begin{aligned}
& \mathrm{PS}_{b}^{\mathrm{PLAN}}\left(t_{\mathrm{DST}}\right)=\mathrm{PS}_{b}^{\mathrm{PLAN}}\left(t_{\mathrm{DST}}\right)+\alpha_{b} \alpha_{b}^{\prime} C, \\
& \mathrm{PD}^{\mathrm{PLAN}}\left(t_{\mathrm{DST}}\right)=\mathrm{PD}^{\mathrm{PLAN}}\left(t_{\mathrm{DST}}\right)+\alpha_{b} \alpha_{b}^{\prime} C .
\end{aligned}
$$

Here, $C$ denotes the shiftable power unit, which is defined as the maximum value satisfying the following constraints:

$$
\begin{aligned}
& \left(\underline{\mathrm{PS}_{b}} \leq-C\right) \wedge\left(\alpha_{b} \alpha_{b}^{\prime} C \leq \overline{\left.\mathrm{PS}_{b}\right)}\right. \text {, } \\
& \forall t \operatorname{ES}_{b}^{\mathrm{PLAN}}(t)<\overline{\mathrm{ES}_{b}} \text {, } \\
& \left(\mathcal{D}\left(t_{\mathrm{SRC}}\right)-\mathrm{PD}^{\mathrm{PLAN}}\left(t_{\mathrm{SRC}}\right)\right)^{2}<\left(\mathcal{D}\left(t_{\mathrm{DST}}\right)-\mathrm{PD}^{\mathrm{PLAN}}\left(t_{\mathrm{DST}}\right)\right)^{2} .
\end{aligned}
$$

If no $C$ satisfies the above constraints, then go to Step 4 . Otherwise, update $\mathcal{T}_{\mathrm{SRC}}=\mathcal{T}_{\mathrm{SRC}}-\left\{t_{\mathrm{SRC}}\right\}$.

If $\mathcal{J}_{\mathrm{SRC}}=\phi$, then go to Step 4. Otherwise return to Step 2 .

[Step 4.]

Update $\mathcal{T}_{\mathrm{DST}}=\mathcal{T}_{\mathrm{DST}}-\left\{t_{\mathrm{DST}}\right\}$.

If $\mathcal{T}_{\mathrm{DST}}=\varnothing$, then terminate. Otherwise, return to Step 1. 
When the algorithm terminates, $\max \operatorname{ES}_{b}^{\text {PLAN }}(t)$ specifies the minimally required capacity of the battery, which could be used to optimize, i.e. reduce, the battery size and cost.

\section{B. Real-Time Demand Mediation and Battery Control}

Among the three real-time processes in the EoD system, the event-driven and periodical re-planning processes should be augmented to manage the battery, while the watch-dog process need not be modified.

\section{1) Feedback control by the periodical re-planning} process

The EoD system controls power consumptions by appliances and power supplies from power sources according to the power usage plan $\operatorname{PD}^{\text {PLAN }}(t)$ and the power supply plan $\operatorname{PS}_{S}^{\text {PLAN }}(t)$ $s \in\{c, b\}$. However, it is difficult to exactly follow the plans because user's life patterns vary day by day. To cope with such variations, the EoD system incorporates the feedback mechanism by the periodical re-planning process.

The periodical re-planning process computes the power supply control target value $T_{s}$ for each power source $s \in\{c, b\}$, with which the event-driven process mediates power consumption demands. At $t=0, T_{s}=\operatorname{PS}_{s}^{\mathrm{PLAN}}(0)$. The periodical re-planning process updates the target value periodically (e.g. every few minutes) based on the accumulated power supplied so far, $E S_{S}\left(t_{\text {now }}\right)$ as follows:

$$
\begin{array}{r}
T_{s}= \begin{cases}\overline{\mathrm{PS}_{s}(t)} & \text { if } T_{s}^{\prime}>\overline{\mathrm{PS}_{s}(t)} \\
\frac{\mathrm{PS}_{s}(t)}{T_{s}^{\prime}} & \text { if } T_{s}^{\prime}<\overline{\mathrm{PS}_{s}(t)} \\
\text { otherwise }\end{cases} \\
T_{s}^{\prime}=T_{s}-\frac{t_{\text {now }}}{T}\left(\mathrm{ES}_{s}\left(t_{\text {now }}\right)-\operatorname{ES}_{s}^{\mathrm{PLAN}}\left(t_{\text {now }}\right)\right),
\end{array}
$$

where $\frac{t_{\text {now }}}{T}$ denotes a feedback gain. While the gain is small in the early stage, it becomes large in the final stage to satisfy the ceiling constraint.

2) Demand-supply mediation by the event-driven process

The event-driven process in the previous EoD system[3] curbs power consumption demands from appliances exactly down below the target value $T_{S}$ based on their associated priority values. While this curbing algorithm works well to comply with the specified restriction and ceiling constraints, a user may feel uncomfortable when he/she wants to use large power, e.g. by coking, earlier than usual. That is, since $T_{s}$ at such earlier time stays low, many power demands are curbed.

To solve this problem, we first introduce the power supply capacity index profile for each power source. Fig. 4 illustrates the one used in the previous EoD system for the commercial utility power source and the augmented one in the new EoD system respectively, where the horizontal axis denotes the power supply and the vertical axis the capacity index. The capacity index lines imply that power consumption demands from appliances whose priority values are smaller than the line are rejected. Note that this demand curbing is not a static process of thresholding demand priorities with the capacity index but a dynamical one to iteratively reject demands and reduce the total power demand at $\mathrm{t}, \mathrm{PD}(\mathrm{t})$ based on the demand priorities so that all remaining demands satisfy the capacity

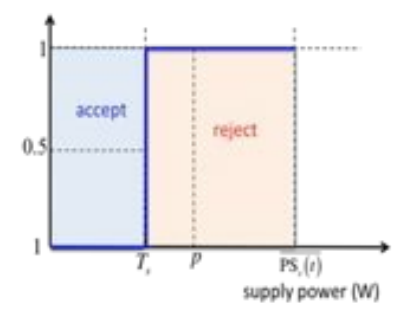

(a) previous EoD

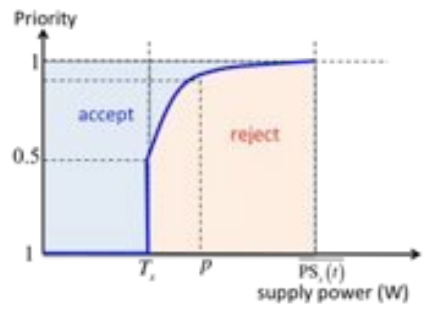

(b) augmented EoD.
Fig. 4 Capacity index profiles for the previous EoD and the augmented EoD..

index profile. In Fig. 4(a), for example, if $\mathrm{PD}(\mathrm{t})>T_{s}$, find the demand with the lowest priority. If it is less than 1 , the demand is rejected or its source appliance is asked to reduce the requested power, which then reduces $\mathrm{PD}(\mathrm{t})$. Iterate this demand curbing process until all remaining demands are placed in the accept area specified by the capacity index profile.

The augmented EoD system employs the soft demand curbing algorithm by introducing the capacity index profile as shown in Fig. 4(b), which enables to accept earlier shifted demand peaks. That is, even if $\mathrm{PD}(\mathrm{t})>T_{s}$, power requests with high priorities can be accepted. In this paper, we define the capacity index function $\mathrm{CI}_{s}(p)$ for power source $s$ as follows:

$$
\mathrm{CI}_{s}(p)=\left\{\begin{array}{cc}
0 & \text { if } p<T_{s} \\
\frac{1}{\pi} \tan ^{-1}\left(\beta_{s} \frac{p-T_{s}}{\overline{\mathrm{ES}_{s}}-\mathrm{ES}_{s}(t)}\right)+0.5 & \text { otherwise; }
\end{array}\right.
$$

where $\boldsymbol{\beta}_{\boldsymbol{s}}$ denotes a control parameter to be specified by a user.

Then the demand-supply mediation algorithm can be defined by using the capacity index function. Let $\mathrm{PD}_{a}^{\mathrm{REQ}}$ and $\operatorname{Pri}_{a}\left(\mathrm{PD}_{a}^{\mathrm{REQ}}\right)$ denote the power requested by appliance $a$ and its priority respectively, and $\mathrm{PS}_{s}^{\mathrm{RES}}$ the power supplied from power source $s$. The appliances and power sources should satisfy following condition:

$$
\forall a \in A, \forall s \in S: \operatorname{Pri}_{a}\left(\mathrm{PD}_{a}^{\mathrm{REQ}}\right) \geq \mathrm{CI}_{s}\left(\mathrm{PS}_{s}^{\mathrm{RES}}\right) .
$$

Since the total power consumptions by all appliances must be equal to the total power supplies from all power sources, $\mathrm{PD}_{a}^{\mathrm{REQ}}$ and $\mathrm{PS}_{s}^{\mathrm{RES}}$ should satisfy following equation;

$$
\sum_{a \in A} \mathrm{PD}_{a}^{\mathrm{REQ}}=\sum_{s \in S} \mathrm{PS}_{S}^{\mathrm{RES}} \text {. }
$$

When the EoD manager receives a new power request message from an appliance, the event-driven process starts the demand-supply mediation by the following algorithm. Let $A$ denote a set of operating and newly power requesting appliances, $\mathrm{PD}_{a}^{\mathrm{REQ}}$ the consuming/requested power of appliance $a \in A, \operatorname{Pri}_{a}(p)$ the priority of the power request $p$ by appliance $a, S=\{b, c\}$ a set of power sources, and $\mathrm{CI}_{s}(p)$ the capacity index function of power source $s$.

\section{[Initialize]}

If $\sum_{a \in A} \mathrm{PD}_{a}^{\mathrm{REQ}}<\sum_{s \in S} T_{s}$, then supply power to the newly requesting appliance and terminate the mediation process. Otherwise, compute the initial value of $\mathrm{PS}_{s}^{\mathrm{RES}}$ to satisfy all demands $\sum_{a \in A} \mathrm{PD}_{a}^{\mathrm{REQ}}$ based on Eq. (27) as follows: 


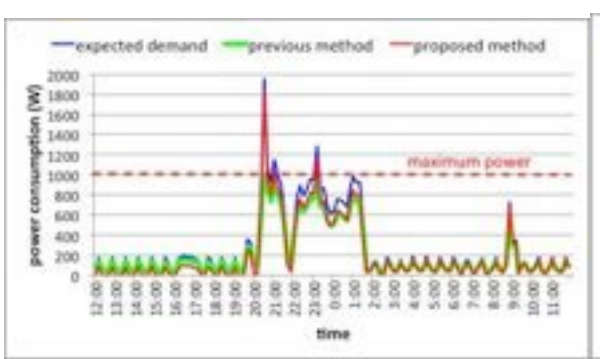

(a) Instantaneous power consumption.

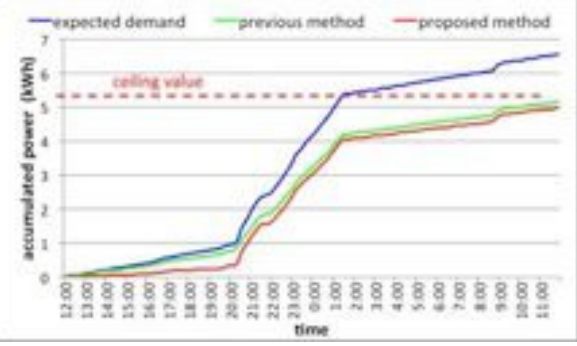

(b) Accumulated power consumption. (c) Power supply pattern for each supplier.

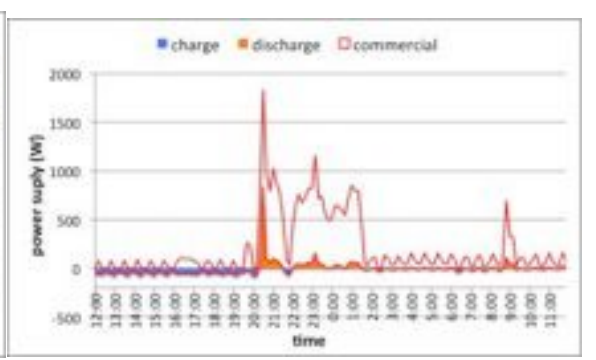
.

$$
\mathrm{PS}_{s}^{\mathrm{RES}}=T_{s}+\frac{1}{|S|}\left(\sum_{a \in A} \mathrm{PD}_{a}^{\mathrm{REQ}}-\sum_{s \in S} T_{s}\right) .
$$

\section{[Step 1.]}

Select appliance $a_{\min } \in A$ with the minimum priority and the power source $s_{\max }$ with the maximum capacity index.

$$
\begin{gathered}
\mathrm{a}_{\min }=\underset{a \in A}{\arg \min } \operatorname{Pri}_{a}\left(\mathrm{PD}_{a}^{\mathrm{REQ}}\right), \\
\mathrm{s}_{\max }=\underset{s \in S}{\arg \max } \mathrm{CI}_{s}\left(\mathrm{PS}_{s}^{\mathrm{RES}}\right) .
\end{gathered}
$$

[Step 2.]

If $\operatorname{Pri}_{a_{\min }}\left(\mathrm{PD}_{a_{\min }}^{\mathrm{REQ}}\right) \geq \mathrm{CI}_{s_{\max }}\left(\mathrm{PS}_{S_{\max }}^{\mathrm{RES}}\right)$, then terminate the mediation process, because the constraint Eq. (26) is satisfied.

\section{[Step 3.]}

Reduce the power for $a_{\text {min }}$ by either turning it off, suspending its operation, or asking $a_{\min }$ to decrease the power consumption depending on its power controllability. Let $\mathrm{PD}_{a_{\min }}^{\mathrm{NEXT}}$ be the reduced power consumption for the $a_{\mathrm{min}}$. Update the supplying power for $s_{\max }$ and request power for $a_{\text {min }}$ as follows:

$$
\begin{aligned}
& \mathrm{PS}_{S_{\max }}^{\mathrm{RES}}:=\mathrm{PS}_{S_{\max }}^{\mathrm{RES}}-\left(\mathrm{PD}_{a_{\min }}^{\mathrm{REQ}}-\mathrm{PD}_{a_{\min }}^{\mathrm{NEXT}}\right), \\
& \mathrm{PD}_{a_{\min }}^{\mathrm{REQ}}:=\mathrm{PD}_{a_{\min }}^{\mathrm{NEXT}}
\end{aligned}
$$

Note that when appliance $a_{\min }$ is turned off or its operation is suspended, its power demand request is eliminated. Note that the suspended appliance waits for a while and issues a new power demand request with a higher priority. When the power consumption is reduced, on the other hand, its corresponding power demand request priority is usually increased. After these modifications, go to Step. 1.

\section{EXPERIMENTS}

\section{A. Experimental Environments}

We developed a smart apartment room equipped with smart taps[4] to demonstrate detailed power consumption visualization in real life environments[5]. The room includes 19 appliances attached with smart taps (ceiling lights, TV, DVD player, electric cooker, air conditioner, hair dryer, etc). Five people lived in the room (three days per person) to monitor and collect power consumption patterns in real world lives.
We conducted simulation experiments using the collected life pattern data, where we assume a person in the room controls the appliances as specified in the data and the EoD system virtually controls the appliances and a storage battery to comply with the restriction and ceiling constraints while minimizing the human demand dissatisfaction.

\section{B. Power Supply and Consumption Planning}

The expected power consumption $\mathcal{D}(t)$ is obtained by the moving average of the collected data. For the commercial utility power source, we set the restriction as $\overline{\mathrm{PS}_{c}(t)}=1000 \mathrm{~W}$ and the ceiling $\overline{\mathrm{ES}_{c}}$ to be $30 \%$ less than the one computed from $\mathcal{D}(t)$. For the storage battery, we set the storage capacity as $\overline{\mathrm{ES}_{b}}=500 \mathrm{Wh}$, the maximum charge and discharge powers as $\mathrm{PS}_{b}=-1000 \mathrm{~W}$ and $\overline{\mathrm{PS}_{b}}=1000 \mathrm{~W}$, respectively, and the

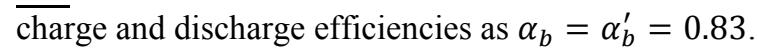

Fig. 5 (a) and (b) illustrate the results by the power supply and consumption planning. The blue lines indicate the expected demand pattern $\mathcal{D}(t)$ and its accumulated version, the green lines the planning results by the previous EoD without a battery, and the red lines by the proposed algorithm. Fig. 5 (c) shows the power supply patterns by the commercial utility power source and the storage battery. We can observe that the storage battery worked well to supply sufficient power during demand peaks while charging during low demand periods. It should be noted that such dynamic battery control is planned based on the pre-learned life pattern $\mathcal{D}(t)$, which differs greatly from one household/office/factory to another. These results prove that the proposed planning algorithm can make full use of the battery to satisfy power request demands as many as possible while keeping the restriction and ceiling constraints, i.e. saving energy, realizing eco-life, and, moreover, complying with the demand response request. While not implemented, the energy cost factor can be easily introduced into the algorithm.

\section{Real-Time Demand Mediation and Battery Control}

Fig. 6 and 7 show the results of the real-time demand mediation by the previous and augmented EoD systems respectively. The blue lines indicate a power demand pattern $\mathcal{D}^{\prime}(t)$, which is different from $\mathcal{D}(t)$ and hence has a highest demand peak one hour earlier than $\mathcal{D}(t)$. The red lines denote the power usage plans generated based on $\mathcal{D}(t)$ and the green lines the mediated power consumption patterns.

The previous EoD system cannot cope with earlier demand peaks and just curbs demands (Fig. 6 (a)). As a result, the accumulated power consumption becomes much less than the planned one (Fig. 6 (b)), which would damage human activities 


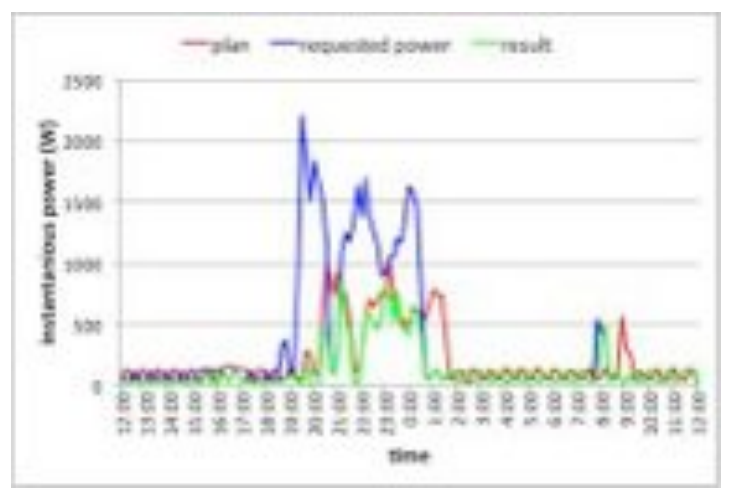

(a) Instantaneous power consumption.

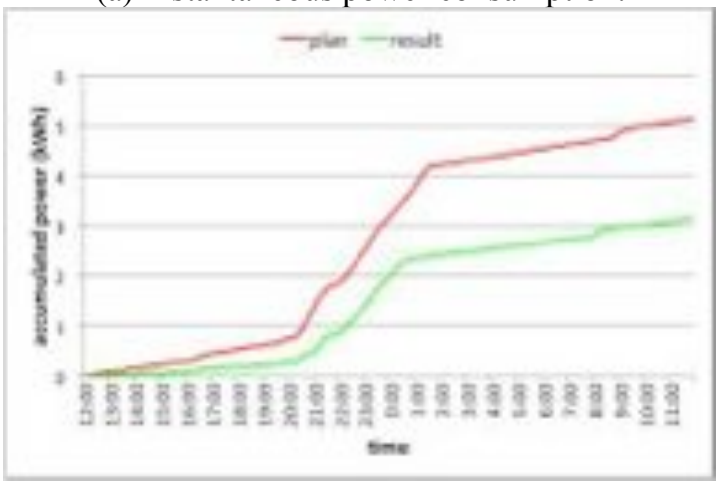

(b) Accumulated energy consumption.

Fig. 6 Real-time demand mediation by the previous EoD system.

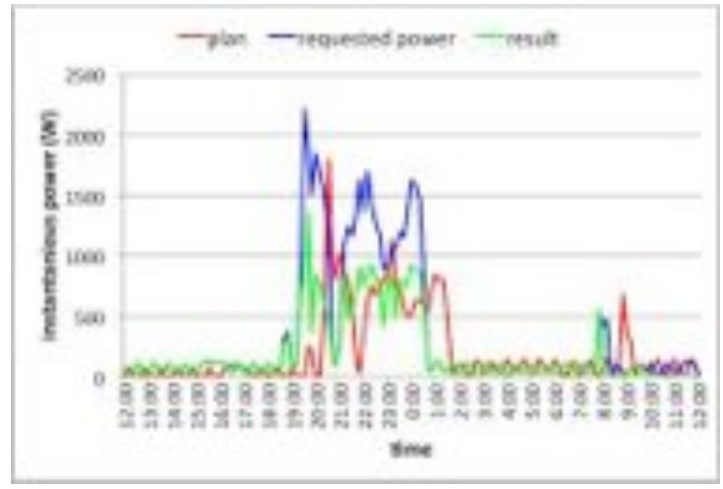

(a) Instantaneous power consumption.

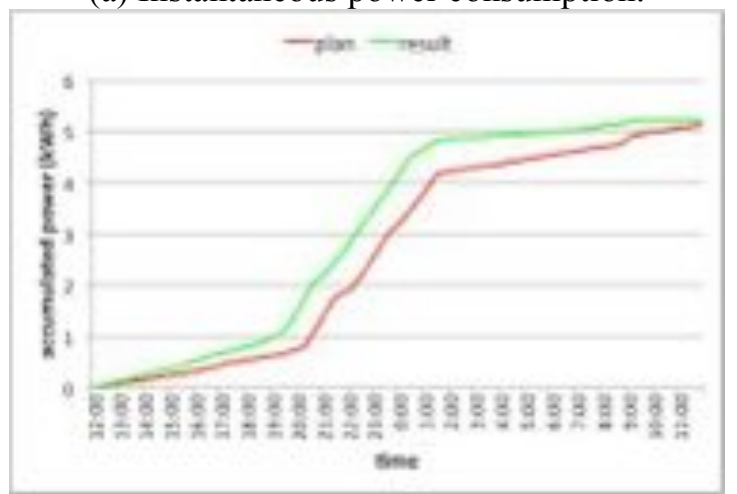

(b) Accumulated energy consumption.

Fig. 7 Real-time demand mediation by the augmented EoD system.

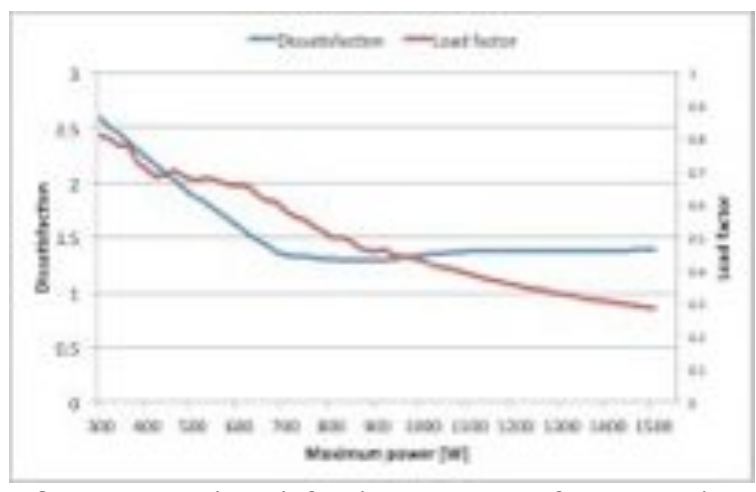

Fig. 8 Demand dissatisfaction and load factor against maximum power of commercial power source.

significantly. Thanks to the improved power supply capacity index profiles for the power sources as well as the battery introduction, the augmented EoD system can cope with earlier demand peaks (Fig. 7 (a)) and can make full use of the usable total power specified by the ceiling (Fig. 7 (b)), which would maximize the comfortability of human activities under the constraints.

Our system can improve fluctuation in commercial power source without damage for user's quality of life. Fig. 8 shows demand dissatisfaction DS(PD) and load factor $\left(\mathrm{LF}_{c}=\right.$ $\left.\frac{\frac{1}{|T|} \sum_{t}^{T} \mathrm{PS}_{c}(t)}{\max _{\mathrm{t} \in \mathrm{T}} \mathrm{PS}_{c}(t)}\right)$ of commercial power source while changing the maximum power $\overline{\mathrm{PS}_{c}(t)}$ for commercial power source from $300 \mathrm{~W}$ to $1500 \mathrm{~W}$. We can see that the load factor can be improved with the low dissatisfaction when the maximum power is larger than $700 \mathrm{~W}$.

\section{CONCLUSION}

This paper proposed a novel adaptive storage battery management mechanism based on the Energy on Demand system and demonstrated its effectiveness with simulations using real-life data. Future works include the implementation of the proposed system in the smart apartment room and its further improvement to manage other distributed power sources: a PV, a fuel cell, and so forth.

\section{ACKNOWLEDGEMENT}

This work was partially supported by JST, CREST and Grant-in-Aid for Scientific Research 23700169.

\section{REFERENCES}

[1] T.Matsuyama,"Creatingsafe,secure,andenvironment-friendlylifestyles hrough i-energy," New Breeze, vol. 21, no. 2, pp. 1-8, 2009.

[2] NIST, "Smart grid home page," http://www.nist.gov/smartgrid/.

[3] T. Kato, K. Yuasa, and T. Matsuyama, "Energy on demand: Efficient and versatile energy control system for home energy management," Proc. of IEEE SmartGridComm2011, Brussels Belugium, pp. 410-415, 2011.

[4] T. Kato, H. Cho, D. Lee, T. Toyomura, and T. Yamazaki. Appliance Recognition from Electric Current Signals for Information-Energy Integrated Network in Home Environments. International Journal of Assistive Robotics and Systems, Vol. 10, No. 4, pp. 51-60, 2009.

[5] "Smart Apartment with an integrated Smart Tap Network," http://www.youtube.com/watch?v=QRQ72xtzDHE, 